\title{
Spike-burst chimera states in an adaptive exponential integrate-and-fire neuronal network
}

Cite as: Chaos 29, 043106 (2019); https://doi.org/10.1063/1.5087129

Submitted: 28 December 2018 . Accepted: 20 March 2019 . Published Online: 10 April 2019

Moises S. Santos (D), Paulo R. Protachevicz (D), Kelly C. larosz (D), lberê L. Caldas (iD, Ricardo L. Viana (D), Fernando S. Borges (D), Hai-Peng Ren (D), José D. Szezech (D), Antonio M. Batista (D), and Celso Grebogi
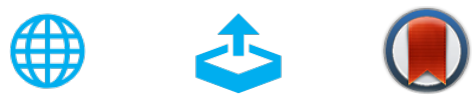

View Online

Export Citation

\section{ARTICLES YOU MAY BE INTERESTED IN}

Clustering and Bellerophon state in Kuramoto model with second-order coupling Chaos: An Interdisciplinary Journal of Nonlinear Science 29, 043102 (2019); https:// doi.org/10.1063/1.5085407

Repulsively coupled Kuramoto-Sakaguchi phase oscillators ensemble subject to common noise

Chaos: An Interdisciplinary Journal of Nonlinear Science 29, 033127 (2019); https:// doi.org/10.1063/1.5084144

Forced synchronization of a multilayer heterogeneous network of chaotic maps in the chimera state mode

Chaos: An Interdisciplinary Journal of Nonlinear Science 29, 033134 (2019); https:// doi.org/10.1063/1.5090184

\section{AlP Author Services English Language Editing}




\title{
Spike-burst chimera states in an adaptive exponential integrate-and-fire neuronal network
}

\author{
Cite as: Chaos 29, 043106 (2019); doi: 10.1063/1.5087129 \\ Submitted: 28 December 2018 . Accepted: 20 March 2019. \\ Published Online: 10 April 2019
}

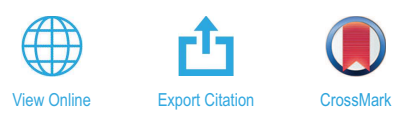

Moises S. Santos, ${ }^{1,2}$ (D) Paulo R. Protachevicz, ${ }^{2}$ (D) Kelly C. larosz, ${ }^{3}$ (D) Iberê L. Caldas, ${ }^{3}$ (D) Ricardo L. Viana, ${ }^{1}$ Fernando S. Borges, ${ }^{4}$ (D) Hai-Peng Ren, ${ }^{5,6}$ (i) José D. Szezech, Jr., ${ }^{2,7}$ (iD) Antonio M. Batista, ${ }^{2,7}$ (D) and Celso Grebogi ${ }^{8}$

\author{
AFFILIATIONS \\ ${ }^{1}$ Department of Physics, Federal University of Paraná, 80060-000 Curitiba, PR, Brazil \\ ${ }^{2}$ Graduate in Science Program-Physics, State University of Ponta Grossa, 84030-900 Ponta Grossa, PR, Brazil \\ ${ }^{3}$ Institute of Physics, University of São Paulo, 05508-900 São Paulo, SP, Brazil \\ ${ }^{4}$ Center for Mathematics Computation and Cognition, Federal University of ABC, 09606-045 São Bernardo do Campo, \\ SP, Brazil \\ ${ }^{5}$ Shaanxi Key Laboratory of Complex System Control and Intelligent Information Processing, Xian University of Technology, \\ Xi'an 710048, People's Republic of China \\ ${ }^{6}$ Xian Technological University, Xi'an 710021, People's Republic of China \\ ${ }^{7}$ Department of Mathematics and Statistics, State University of Ponta Grossa, 84030-900 Ponta Grossa, PR, Brazil \\ ${ }^{8}$ Institute for Complex Systems and Mathematical Biology, King's College, University of Aberdeen, Aberdeen AB24 $3 U E$, \\ United Kingdom
}

Note: This paper is part of the Focus Issue, "Nonlinear Chemical Dynamics and Its Interdisciplinary Impact: Dedicated to Ken Showalter on the Occasion of his 70th Birthday."

\begin{abstract}
Chimera states are spatiotemporal patterns in which coherence and incoherence coexist. We observe the coexistence of synchronous (coherent) and desynchronous (incoherent) domains in a neuronal network. The network is composed of coupled adaptive exponential integrateand-fire neurons that are connected by means of chemical synapses. In our neuronal network, the chimera states exhibit spatial structures both with spike and burst activities. Furthermore, those desynchronized domains not only have either spike or burst activity, but we show that the structures switch between spikes and bursts as the time evolves. Moreover, we verify the existence of multicluster chimera states.
\end{abstract}

Published under license by AIP Publishing. https://doi.org/10.1063/1.5087129

Many coupled dynamical systems exhibit complex spatiotemporal patterns characterized by coexistence of coherence and incoherence regions. These patterns were observed in chemical systems, mechanical oscillator networks, and electronic circuits. Neuronal systems can exhibit coexisting domains of coherence and incoherence behavior. We build a network composed of adaptive exponential integrate-and-fire neurons. This neuron model mimics the dynamics of the membrane potential. Our neuronal network has neurons that can display spiking or bursting activities. We identify chimera states with spatiotemporal domains separated into synchronous and desynchronous behaviors. In addition, we show the presence of chimera states whose neurons switch in the time between spikes and bursts.

\section{INTRODUCTION}

In dynamical systems, the word chimera has been used to describe the coexistence of coherent and incoherent patterns. ${ }^{1,2}$ Chimera states were first observed by Umberger et al. in $1989 .^{3}$ Kuramoto and Battogtokh ${ }^{4}$ reported spatiotemporal patterns of coexisting coherence and incoherence in nonlocally coupled phase oscillators. ${ }^{5}$

There are experimental evidences of chimera states in coupled chaotic optoelectronic oscillators, ${ }^{6}$ mechanical systems, ${ }^{7,8}$ network of electrochemical reactions, ${ }^{9}$ and populations of coupled chemical oscillators. ${ }^{10,11}$ Totz et al. ${ }^{12}$ reported the existence of spiral wave chimera states in coupled chemical oscillators. They carried out experiments with coupled Belousov-Zhabotinsky chemical oscillators. 
Numerical analysis of coupled systems has showed the coexistence of coherent and incoherent domains ${ }^{13,14}$ and basin riddling in chimera dynamics. ${ }^{15}$ Chimera states were found in simulations of different neuronal networks, e.g., coupled Hindmarsh-Rose neurons, ${ }^{16,17}$ coupled FitzHugh-Nagumo neurons, ${ }^{18,19}$ coupled Hodgkin-Huxley neurons, ${ }^{20,21}$ network of integrate-and-fire neurons, ${ }^{22,23}$ and network composed of heterogeneous neurons. ${ }^{2}$ Rothkegel and Lehnertz ${ }^{25}$ found chimera states in small-world networks of excitatory integrate-and-fire-like models. ${ }^{26}$ Hizanidis et al. ${ }^{27}$ observed synchronous, metastable chimera states in a modular organization of the $C$. elegans neuronal network. Ren et al. ${ }^{28}$ showed the coexistence of different periodic states in the Hindmarsh-Rose neuron network with both chemical and electrical connections. Santos et al..$^{29}$ reported the presence of chimeras in the neuronal networks. They considered a network model based on the cat cerebral cortex and identified two different chimera patterns characterized by desynchronized spikes and bursts. In the human brain, there are analogies between chimera state collapses and epileptic seizures. ${ }^{30}$

In this paper, we study a network of adaptive exponential integrate-and-fire neurons. ${ }^{31}$ Brette and Gerstner ${ }^{32}$ introduced the adaptive exponential integrate-and-fire (AEIF) as a simple model that mimics the membrane potential of the neuron in vivo. Our neuronal network is a ring of coupled AEIF, in which the neurons are connected by chemical synapses. We observe the existence of chimera states with desynchronized spikes or bursts. The main novelty of our work is to show chimera states whose neurons change between spike and burst activities as the system evolves. In addition, we observe multicluster chimera states that were found by Yao et al. ${ }^{33}$ in Kuramoto networks of phase coupled oscillators. In our neuronal network, the multicluster chimera states are composed not only of temporal changes between spikes and bursts but also of domains with spike and burst patterns.

This paper is organized as follows: Sec. II introduces the neuronal network model. In Sec. III, we show and analyze the time evolution of chimera states in our neuronal network. In Sec. IV, we draw our conclusions.

\section{ADAPTIVE EXPONENTIAL INTEGRATE-AND-FIRE NEURONAL NETWORK}

We build a network composed of $N$ coupled AEIF neurons. The neuron $i$ is symmetrically connected with $R$ nearest neighbors on either side. The neuronal network is composed of adaptive exponential integrate-and-fire neurons, and it is given by

$$
\begin{aligned}
C_{\mathrm{m}} \frac{d V_{i}}{d t}= & -g_{\mathrm{L}}\left(V_{i}-E_{\mathrm{L}}\right)+g_{\mathrm{L}} \Delta_{\mathrm{T}} \exp \left(\frac{V_{i}-V_{\mathrm{T}}}{\Delta_{\mathrm{T}}}\right) \\
& -w_{i}+I_{i}+\left(V_{\mathrm{REV}}-V_{i}\right) \sum_{j=i-R, j \neq i}^{i+R} g_{j}, \\
\tau_{w} \frac{d w_{i}}{d t}= & a\left(V_{i}-E_{\mathrm{L}}\right)-w_{i}, \\
\tau_{\mathrm{s}} \frac{d g_{i}}{d t}= & -g_{i},
\end{aligned}
$$

where $V_{i}$ is the membrane potential, $w_{i}$ is the adaptation current, and $g_{i}$ is the synaptic conductance. We consider membrane capacitance $C_{\mathrm{m}}=200 \mathrm{pF}$, resting potential $E_{\mathrm{L}}=-70 \mathrm{mV}$, leak conductance $g_{\mathrm{L}}=12 \mathrm{nS}$, slope factor $\Delta_{\mathrm{T}}=2 \mathrm{mV}$, spike threshold potential $V_{\mathrm{T}}=-50 \mathrm{mV}$, adaptation time constant $\tau_{w}=300 \mathrm{~ms}$, level of subthreshold adaptation $a=2 \mathrm{nS}$, synaptic time constant $\tau_{\mathrm{s}}=2.728 \mathrm{~ms}$, injection of current $I_{i}=500 \mathrm{pA}$, and synaptic reversal potential $V_{\mathrm{REV}}=0 \mathrm{mV}$ (excitatory synapses). When $V_{i}$ is larger than a threshold, $V_{i}>V_{\text {thres }},{ }^{34} V_{i}, w_{i}$, and $g_{i}$ are updated following the rules

$$
\begin{aligned}
V_{i} & \rightarrow V_{\mathrm{r}}, \\
w_{i} & \rightarrow w_{i}+b, \\
g_{i} & \rightarrow g_{i}+g_{\mathrm{ex}},
\end{aligned}
$$

where $V_{\mathrm{r}}=-58 \mathrm{mV}, b=70 \mathrm{pA}$, and $g_{\text {exc }}$ is the intensity of the excitatory synaptic conductance.

Depending on the control parameters, the AEIF neurons can generate spike or burst firings, and the network can also exhibit synchronous and desynchronous behavior. In all simulations, we consider that the individual uncoupled neurons perform spike activities for the chosen parameters. With regard to the initial conditions, $V_{i}$ and $w_{i}$ are randomly distributed in the intervals $[-58,-43] \mathrm{mV}$ and $[0,70] \mathrm{pA}$, respectively. We analyze the solution of the neuronal network model during $2 \mathrm{~s}$ and discard a transient time equal to $4 \mathrm{~s}$.

To identify spike or burst activities, we calculate the coefficient of variation $\left(\mathrm{CV}_{i}\right)$,

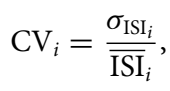

where $\overline{\mathrm{ISI}}_{i}$ is the mean value of the time difference between two consecutive firings (interspike interval) of the neuron $i$ and $\sigma_{\mathrm{ISI}_{i}}$ is the standard deviation of $\operatorname{ISI}_{i}$. In Fig. 1, we see the action potential of the neuron $i$ for (a) spikes $\left(\mathrm{CV}_{i}=0.008\right)$, (b) spikes and bursts $\left(\mathrm{CV}_{i}=0.529\right)$, and (c) bursts $\left(\mathrm{CV}_{i}=0.833\right)$.

The mean value of $\mathrm{CV}(\overline{\mathrm{CV}})$ is given by

$$
\overline{\mathrm{CV}}=\frac{1}{N} \sum_{i=1}^{N} \mathrm{CV}_{i} .
$$

\section{(a)}

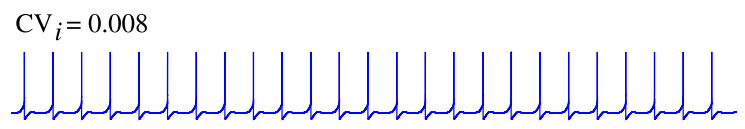

(b) $\mathrm{CV}_{i}=0.529$

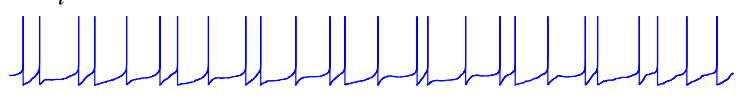

(c) $\mathrm{CV}_{i}=0.833$

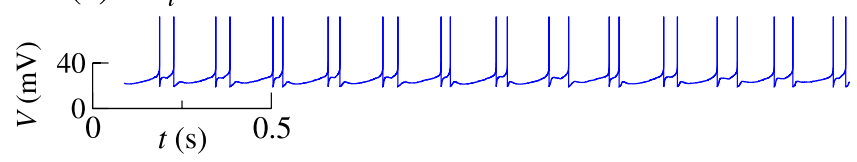

FIG. 1. Action potential of the neuron $i$ in our neuronal network for (a) spikes $\left(\mathrm{CV}_{i}=0.008\right)$, (b) spikes and bursts $\left(\mathrm{CV}_{i}=0.529\right)$, and (c) bursts $\left(\mathrm{CV}_{i}=\right.$ 0.833). 


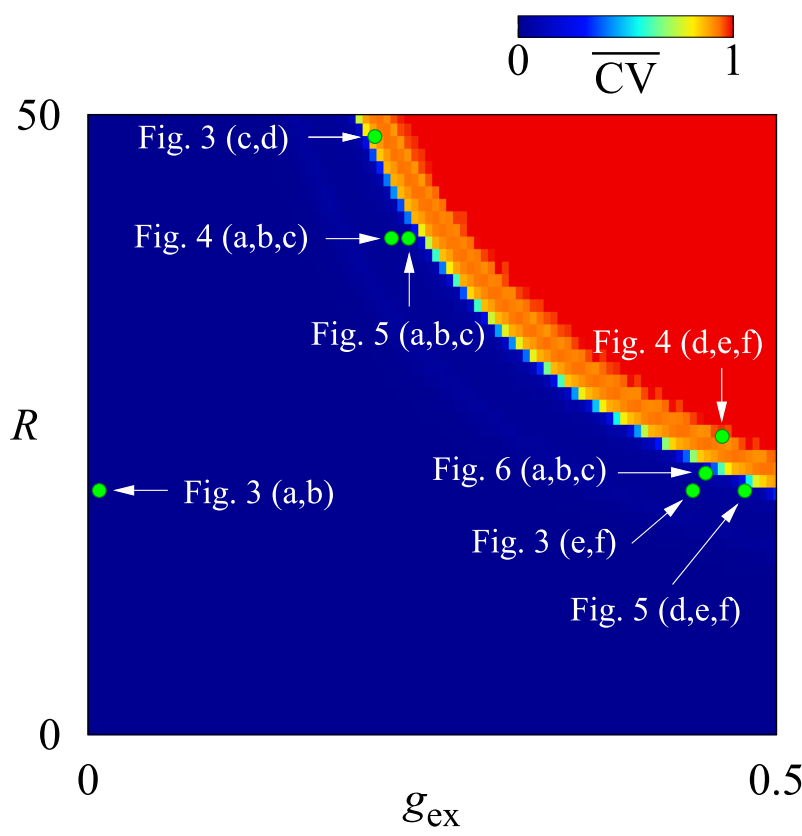

FIG. 2. Parameter space $R \times g_{\mathrm{ex}}$ for $\overline{\mathrm{CV}}$, where we consider $N=1000$ coupled $\mathrm{AEIF}$ neurons and 50 different initial conditions. The regions have spiking $(\overline{\mathrm{CV}}<$ $0.5)$, bursting ( $\overline{\mathrm{CV}} \geq 0.5)$ neurons, and the coexistence of bursting and spiking can be seen according to the color.

For $\overline{\mathrm{CV}}<0.5$ and $\overline{\mathrm{CV}} \geq 0.5$, the neuronal network exhibits spikes and bursts, respectively. ${ }^{35}$ Figure 2 shows the parameter space $R \times g_{\text {ex }}$ for $\overline{\mathrm{CV}}$ in color scale. The regions for $\overline{\mathrm{CV}}<0.5$ and $\overline{\mathrm{CV}} \geq 0.5$ correspond to spike and burst activities, respectively. In the transition region, we identify the coexistence of spike and burst behaviors for different neurons in the network. Synchronous and desynchronous behaviors also occur for different values as $R$ and $g_{\mathrm{ex}}$.

\section{CHIMERA STATES}

In the chimera states, there are spatiotemporal patterns characterized by the coexistence of coherent and incoherent domains. The spatial coherence and incoherence can be identified by the local order parameter $^{36}$

$$
Z_{j}(t)=\left|\frac{1}{2 \delta+1} \sum_{|j-k| \leq \delta} \mathrm{e}^{\mathrm{i} \phi_{k}(t)}\right|, \quad k=1, \ldots, N .
$$

The phase is defined as

$$
\phi_{k}(t)=2 \pi m+2 \pi \frac{t-t_{k, m}}{t_{k, m+1}-t_{k, m}}
$$

where $t_{k, m}$ is the time of the $m$ th spike of the neuron $k, t_{k, m}<t$ $<t_{k, m+1}$, and the spike happens for $V_{k}>V_{\text {thres }}$. In our simulations,
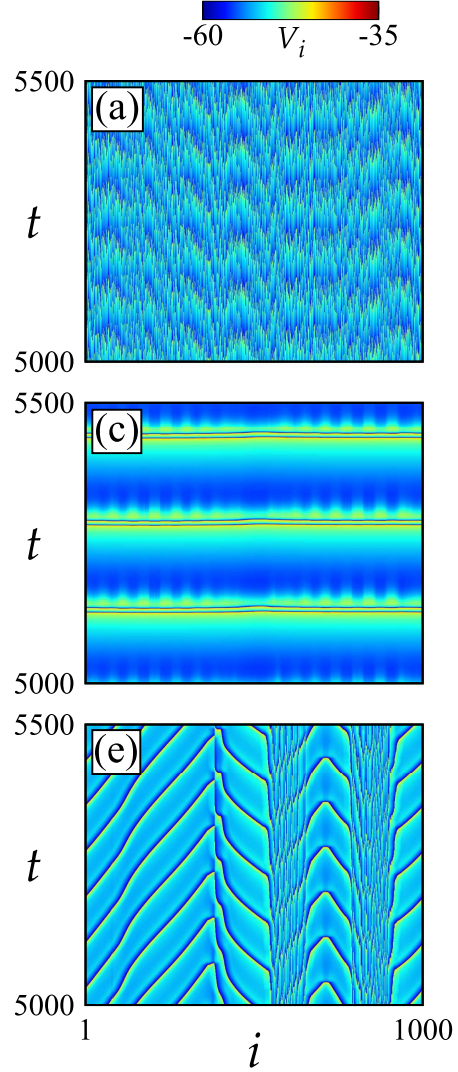

1000
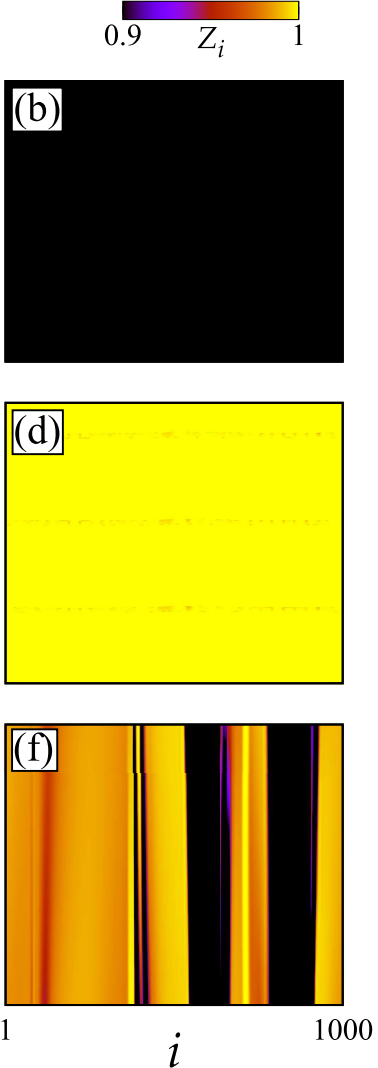

FIG. 3. Time evolution of $V_{i}$ and $Z_{i}$ of each neuron $i$ for incoherent pattern for $R=20$ and $g_{\mathrm{ex}}=0.01 \mathrm{nS}[(\mathrm{a})$ and (b)], synchronized behavior for $R=48$ and $g_{\mathrm{ex}}=0.21 \mathrm{nS}[(\mathrm{c})$ and $(\mathrm{d})]$, and chimera state for $R=20$ and $g_{\mathrm{ex}}=0.44 \mathrm{nS}$ $[(e)$ and $(f)]$.

we use $\delta=5$ and consider a pattern to be synchronized when $Z_{j}(t)>$ 0.9. The coherent (synchronized) and incoherent (desynchronized) domains are identified with a minimum size equal to $2 \delta+1$ neighbors.

In Fig. 3, we present different types of dynamic behavior by varying the parameters $R$ and $g_{\text {ex }}$. Figures 3(a) and 3(b) display the neuronal network having an incoherent pattern. Depending on the parameters, the network can exhibit synchronization [Figs. 3(c) and $3(\mathrm{~d})$ ]. We find chimera states with coexisting synchronous and desynchronous domains, as shown in Figs. 3(e) and 3(f).

Our AEIF neuronal network can exhibit different firing patterns, such as spikes, bursts, or both spikes and bursts. Figure 4 exhibits the values of $V_{i}, Z_{i}$, and $\mathrm{CV}_{i}$ for chimera with spiking neurons [Figs. 4(a) $-4(\mathrm{c})$ ] and chimera with bursting neurons [Figs. 4(d) $-4(f)]$.

A phenomenon not seen before is that the chimera states keep switching, in the desynchronous domains, between spikes and bursts as the system evolves in time, which we call spike-burst chimera (SBC). The SBC is found through $Z_{j}(t)$ and $\mathrm{CV}_{i}$. We identify SBC 

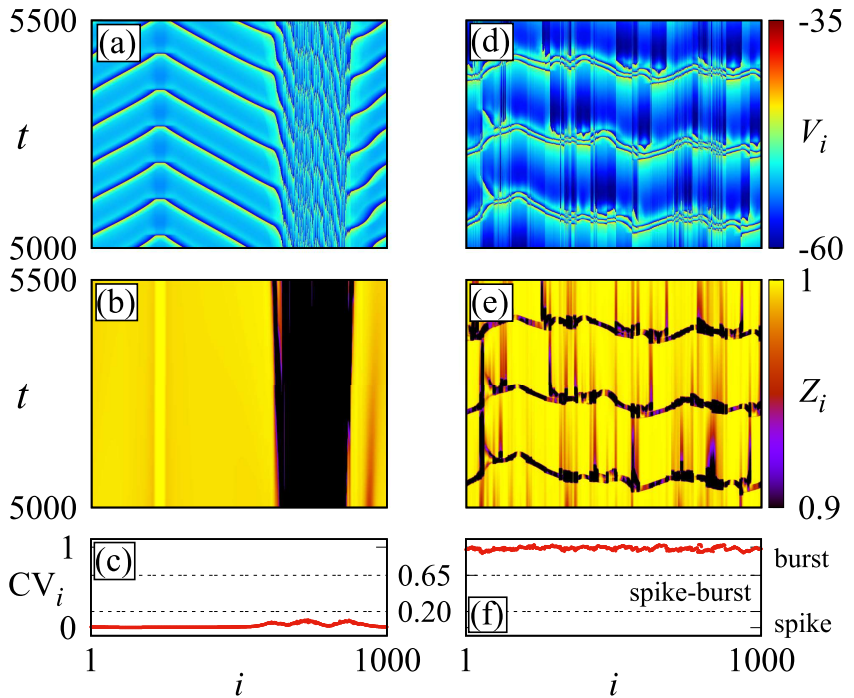

FIG. 4. $V_{i}$ and $Z_{i}$ in color scale for $t \times i$ and $C_{i} \times i$. Chimera with spiking neurons for $R=40$ and $g_{\mathrm{ex}}=0.22 \mathrm{nS}[(\mathrm{a})-(\mathrm{c})]$, and chimera with bursting neurons for $R=24$ and $g_{\mathrm{ex}}=0.46372 \mathrm{nS}[(\mathrm{d})-(\mathrm{f})]$.

when the $\mathrm{CV}_{i}$ values are in the interval $[0.2,0.65]$. In Fig. 5, we calculate $V_{i}, Z_{i}$, and $\mathrm{CV}_{i}$ for parameters when the $\mathrm{SBC}$ states are present. Figures 5(a)-5(c) display a SBC with synchronized spikes for $R=40$ and $g_{\mathrm{ex}}=0.233 \mathrm{nS}$. The SBC can have synchronized bursts, as shown in Figs. 5(d)-5(f) for $R=20$ and $g_{\text {ex }}=0.48 \mathrm{nS}$.
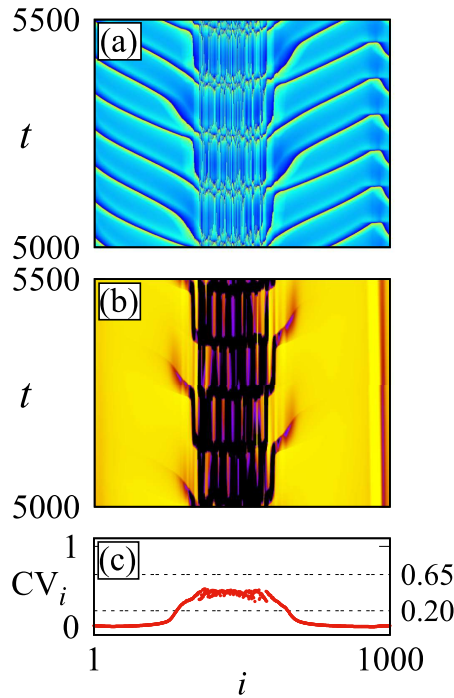
0.20 20
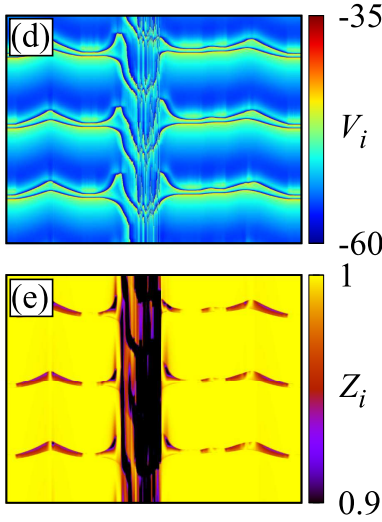

FIG. 5. $V_{i}$ and $Z_{i}$ in color scale for $t \times i$ and $\mathrm{CV}_{i} \times i$. The SBC with synchronized spikes for $R=40$ and $g_{\mathrm{ex}}=0.233 \mathrm{nS}[(\mathrm{a})-(\mathrm{c})]$, and synchronized bursts for $R=20$ and $g_{\mathrm{ex}}=0.48 \mathrm{nS}[(\mathrm{d})-(\mathrm{f})]$.

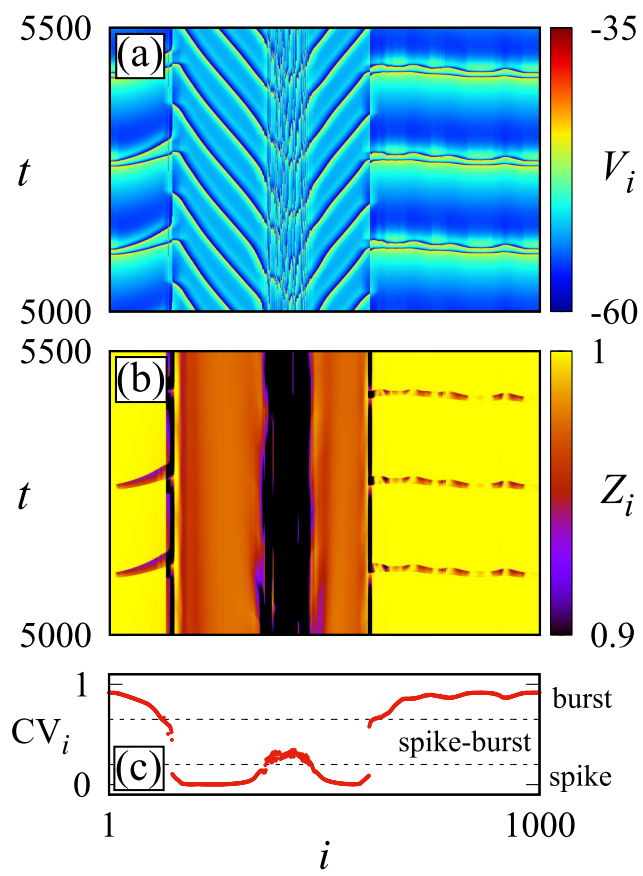

FIG. 6. (a) $V_{i}$, (b) $Z_{i}$ in color scale for $t \times i$, and (c) $C V_{i} \times i$. The figure exhibits multicluster chimera states, where we consider $R=21$ and $g_{\mathrm{ex}}=0.45 \mathrm{nS}$.

In Fig. 6, we show the presence of not only SBC, but also multicluster chimera states. ${ }^{33}$ The network has groups of neurons with different patterns, such as spikes $\left(\mathrm{CV}_{i} \leq 0.20\right)$, bursts $\mathrm{CV}_{i} \geq$ $0.65)$, and a mixture of spikes and bursts $\left(0.20<\mathrm{CV}_{i}<0.65\right)$. Beurrier et $a l .^{37}$ reported that the transition from spike to mixed burst activities in subthalamic nucleus neurons of rat and primates is one of the features of Parkinson's disease. There are two groups with spiking neurons, where one group has 217 neurons and the other has 134 neurons, as well as one group with 511 bursting neurons. The SBC is identified by means of three groups with 22,105 , and 11 neurons that change between spike and burst patterns over time.

Figure 7 (a) shows in the parameter space $R \times g_{\text {ex }}$ the regions for chimera state (CS) and spike-burst chimera (SBC) states, as well as the regions where there are no chimera states (NC). We perform an average of 50 different random initial conditions to compute each point in the parameter space. The regions in blue and white colors represent CS and NC, respectively. The small region in red color denotes the values of $R$ and $g_{\text {ex }}$ in which SBC is observed. Figure 7(b) displays the values of $\overline{C V}$ as a function of $g_{\text {ex }}$ for $R=25$. We observe that $\overline{\mathrm{CV}}$ increases in the transitions from NC to CS and from CS to $\mathrm{SBC}$, showing a scenario from spike to burst activities. When we fix $g_{\text {ex }}$ and vary $R$, we find a similar behavior. There are two disjoint regions with chimera states (CSs) in Fig. 7(a), where region II has a standard deviation of ISI greater than that of region I, consequently, $\overline{\mathrm{CV}}$ in region II is greater than that in region I, as shown in Fig. 7(b). 

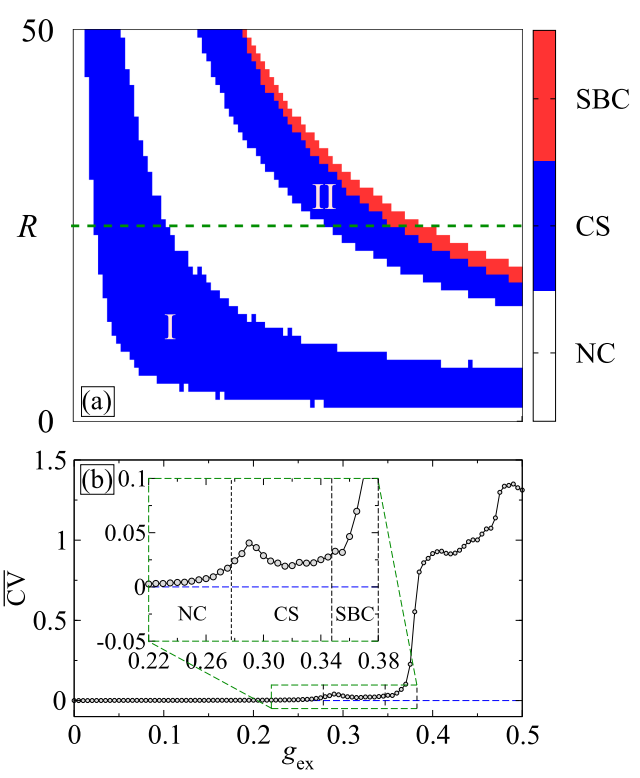

FIG. 7. (a) Parameter space $R \times g_{\mathrm{ex}}$ showing regions with chimera (CS) and spike-burst chimera (SBC), as well as without chimera (NC), where we consider $N=1000$ coupled AEIF neurons and 50 different random initial conditions. (b) $\overline{\mathrm{CV}}$ as a function of $g_{\mathrm{ex}}$ for $R=25$ [green dashed line in Fig. $7(\mathrm{a})$ ].

\section{CONCLUSIONS}

We study an adaptive exponential integrate-and-fire neuronal network. In the network, each neuron is symmetrically coupled to the nearest neighbors. The connectivity between the neurons is given by excitatory synapses. Depending on the control parameters, the neurons can exhibit spike or burst activities.

Research studies have reported the coexistence of spatiotemporal patterns, known as chimera states. There are evidences of chimera states in the brain, e.g., unihemispheric slow-wave sleep in some mammals. The coexistence of synchronous and desynchronous domains has been observed in neuronal network models.

In our network, chimera states are found by varying the number of nearest neighbors and the excitatory synaptic conductance. Depending on the coupling strength, multichimera state can arise for small $R$ values. ${ }^{38}$ We verify the existence of different types of chimera states according to the spike and burst patterns. In this work, we show the existence of chimera states with neurons that change between spike and burst activities as the system evolves in time. Moreover, we also identify multicluster chimera states composed of different groups of neurons with spike and burst patterns, as well as spikes and bursts changing over time.

\section{ACKNOWLEDGMENTS}

We wish to acknowledge the support from Fundação Araucária, CNPq (Grant No. 150701/2018-7), CAPES, and FAPESP (Grant Nos. 2015/07311-7, 2018/03211-6, and 2017/18977-1).

\section{REFERENCES}

${ }^{1}$ I. Omelchenko, Y. Maistrenko, P. Hövel, and E. Schöll, "Loss of coherence in dynamical networks: Spatial chaos and chimera states," Phys. Rev. Lett. 106, 234102 (2011).

${ }^{2} \mathrm{M}$. Wildie and M. Shanahan, "Metastability and chimera states in modular delay and pulse-coupled oscillator networks," Chaos 22, 043131 (2012).

${ }^{3}$ D. K. Umberger, C. Grebogi, E. Ott, and B. Afeyan, "Spatiotemporal dynamics in a dispersively coupled chain of nonlinear oscillators," Phys. Rev. A 39, 4835-4842 (1989).

${ }^{4}$ Y. Kuramoto and D. Battogtokh, "Coexistence of coherence and incoherence in nonlocally coupled phase oscillators," Nonlin. Phenom. Complex Syst. 5, 380-385 (2002).

${ }^{5}$ D. M. Abrams and S. H. Strogatz, "Chimera states for coupled oscillators," Phys. Rev. Lett. 93, 174102 (2004).

${ }^{6}$ J. D. Hart, K. Bansal, T. E. Murphy, and R. Roy, "Experimental observation of chimera and cluster states in a minimal globally coupled network," Chaos 26, 094801 (2016).

${ }^{7}$ E. A. Martens, S. Thutupalli, A. Fourriére, and O. Hallatschek, "Chimera states in mechanical oscillator networks," Proc. Natl. Acad. Sci. 110, 10563-10567 (2013).

${ }^{8}$ T. Kapitaniak, P. Kuzma, J. Wojewoda, K. Czolczynski, and Y. Maistrenko, "Imperfect chimera states for coupled pendula," Sci. Rep. 4, 6379 (2014).

${ }^{9} \mathrm{M}$. Wickramasinghe and I. Z. Kiss, "Spatially organized partial synchronization through the chimera mechanism in a network of electrochemical reactions," Phys, Chem. Chem. Phys. 16, 18360-18369 (2014).

${ }^{10}$ M. R. Tinsley, S. Nkomo, and K. Showalter, "Chimera and phase-cluster states in populations of coupled chemical oscillators,” Nat. Phys. 8, 662-665 (2012).

${ }^{11}$ S. Nkomo, M. R. Tinsley, and K. Showalter, "Chimera states in populations of nonlocally coupled chemical oscillators,” Phys. Rev. Lett. 110, 244102 (2013).

${ }^{12}$ J. F. Totz, J. Rode, M. R. Tinsley, K. Showalter, and H. Engel, "Spiral wave chimera states in large populations of coupled chemical oscillators," Nat. Phys. 14, 282-285 (2018).

${ }^{13}$ O. E. Omel'chenko, Y. L. Maistrenko, and P. A. Tass, "Chimera states: The natural link between coherence and incoherence,” Phys. Rev. Lett. 100, 044105 (2008).

${ }^{14}$ M. S. Santos, J. D. Szezech, Jr, A. M. Batista, I. L. Caldas, R. L. Viana, and S. R. Lopes, "Recurrence quantification analysis of chimera states," Phys. Lett. A 379, 2188-2192 (2015).

${ }^{15}$ V. Santos, J. D. Szezech, Jr, A. M. Batista, K. C. Iarosz, M. S. Baptista, H. P. Ren, C. Grebogi, R. L. Viana, I. L. Caldas, Y. L. Maistrenko, and J. Kurths, "Ridding: Chimera's dilemma," Chaos 28, 081105 (2018).

${ }^{16}$ J. Hizanidis, V. G. Kanas, A. Bezerianos, and T. Bountis, "Chimera states in networks of nonlocally coupled Hindmarsh-Rose neuron models," Int. J. Bifurcation Chaos 24, 1450030 (2014).

${ }^{17}$ B. K. Bera, D. Ghosh, and M. Lakshmanan, "Chimera states in bursting neurons," Phys. Rev. E 93, 012205 (2016).

${ }^{18}$ I. Omelchenko, A. Provata, J. Hizanidis, E. Schöll, and P. Hövel, "Robustness of chimera states for coupled FitzHugh-Nagumo oscillators,” Phys. Rev. E 91, 022917 (2015).

${ }^{19}$ T. Chouzouris, I. Omelchenko, A. Zakharova, J. Hlinka, P. Jiruska, and E. Schöll, "Chimera states in brain networks: Empirical neural vs. modular fractal connectivity," Chaos 28, 045112 (2018).

${ }^{20} \mathrm{H}$. Sakaguchi, "Instability of synchronized motion in nonlocally coupled neural oscillators," Phys. Rev. E 73, 031907 (2006).

${ }^{21}$ T. A. Glaze, S. Lewis, and S. Bahar, "Chimera states in a Hodgkin-Huxley model of thermally sensitive neurons," Chaos 26, 083119 (2016).

${ }^{22}$ C. R. Laing and C. C. Chow, "Stationary bumps in networks of spiking neurons," Neural Comput. 13, 1473-1494 (2001).

${ }^{23}$ N. D. Tsigkri-DeSmedt, J. Hizanidis, E. Schöll, P. Hövel, and A. Provata, "Chimeras in leaky integrate-and-fire neural networks: Effects of reflecting connectivities,” Eur. Phys. J. B 90, 139 (2017).

${ }^{24}$ C. R. Laing, "Bumps in small-world networks," Front. Comput. Neurosci. 10, 53 (2016).

${ }^{25}$ A. Rothkegel and K. Lehnertz, "Irregular macroscopic dynamics due to chimera states in small-world networks of pulsed-coupled oscillators," New J. Phys. 16, 055006 (2014).

${ }^{26}$ A. Rothkegel and K. Lehnertz, "Recurrent events of synchrony in complex networks of pulse-coupled oscillators," Europhys. Lett. 95, 38001 (2011). 
${ }^{27}$ J. Hizanidis, N. E. Kouvaris, G. Zamora-López, C. A. Díaz-Guilera, G. Antonopoulos, "Chimera-like states in modular neural networks," Sci. Rep. 6, 19845 (2016).

${ }^{28}$ H.-P. Ren, C. Bai, M. S. Baptista, and C. Grebogi, "Weak connection forms an infinite number of patterns in the brain," Sci. Rep. 7, 46472 (2017).

${ }^{29}$ M. S. Santos, J. D. Szezech, F. S. Borges, K. C. Iarosz, I. L. Caldas, A. M. Batista, R. L. Viana, and J. Kurths, "Chimera-like states in a neuronal network model of the cat brain,” Chaos Soliton. Fract. 101, 86-91 (2017).

${ }^{30}$ R. G. Andrzejak, C. Rummel, F. Mormann, and K. Schindler, "All together now: Analogies between chimera state collapses and epileptic seizures," Sci. Rep. 6, 23000 (2016).

${ }^{31}$ F. S. Borges, P. R. Protachevicz, E. L. Lameu, R. C. Bonetti, K. C. Iarosz, I. L. Caldas, M. S. Baptista, and A. M. Batista, "Synchronised firing patterns in a random network of adaptive exponential integrate-and-fire neuron model," Neural Netw. 90, 1-7 (2017).

${ }^{32} \mathrm{R}$. Brette and W. Gerstner, "Adaptive exponential integrate-and-fire model as an effective description of neuronal activity," J. Neurophysiol. 94, 3637-3642 (2005).
${ }^{33}$ N. Yao, Z.-G. Huang, C. Grebogi, and Y.-C. Lai, "Emergence of multicluster chimera states," Sci. Rep. 5, 12988 (2015).

${ }^{34}$ R. Naud, N. Marcille, C. Clopath, and W. Gerstner, "Firing patterns in the adaptive exponential integrate-and-fire model," Biol. Cybern. 99, 335-347 (2008).

${ }^{35}$ P. R. Protachevicz, R. R. Borges, A. S. Reis, F. S. Borges, K. C. Iarosz, I. L. Caldas,

E. L. Lameu, E. E. N. Macau, R. L. Viana, I. M. Sokolov, F. A. S. Ferrari, J. Kurths, A. M. Batista, C.-Y. Lo, Y. He, and C.-P. Lin, "Synchronous behaviour in network model based on human cortico-cortical connections," Physiol. Meas. 39, 074006 (2018).

${ }^{36}$ Y. Kuramoto, Chemical Oscillations, Waves, and Turbulence (Springer-Verlag, Berlin, 1984).

${ }^{37}$ C. Beurrier, P. Congar, B. Bioulac, and C. Hammond, "Subthalamic nucleus neurons switch from single-spike activity to burst-firing mode," J. Neurosci. 19 599-609 (1999).

${ }^{38}$ I. Omelchenko, O. E. Omel'chenko, P. Hövel, and E. Schöll, "When nonlocal coupling between oscillators becomes stronger: patched synchrony or multichimera states," Phys. Rev. Lett. 110, 224101 (2013). 